\section{Sobrevida em cinco anos e fatores prognósticos em mulheres com câncer de mama em Santa Catarina, Brasil}

\author{
Five-year survival and prognostic factors in women \\ with breast cancer in Santa Catarina State, Brazil
}

\begin{abstract}
The aim of this study was to analyze breast cancer survival and associated factors, based on a historical cohort of women with breast cancer diagnosis from 2000 to 2002 recorded in the hospital cancer registries at the Santa Catarina Center for Cancer Research and the Irmandade Nosso Senhor dos Passos Charity Hospital, both located in Florianópolis, Santa Catarina State, Brazil. The statistical analysis used the Kaplan-Meier estimator and Cox model. Overall five-year survival was $76.2 \%$ (95\%CI: 73.6-78.9). Independent factors associated with increased risk of death were age less than 30 years (hazard ratio $-H R=3.09$; 95\%CI: 1.25-7.67); illiteracy ( $H R=3.70 ; 95 \% C I$ : 1.44-9.55); and stages III ( $H R=5.27 ; 95 \% C I$ : 2.56-10.82) and $I V(H R=14.07 ; 95 \% C I: 6.81$ 29.06). Young women had the worst survival rates. There were also many women diagnosed in advanced stages, with worse survival, thus demonstrating the need for early diagnostic measures.
\end{abstract}

Breast Neoplasms; Hospital Records; Survival Analysis; Women's Health
Ione Jayce Ceola Schneider Eleonora d'Orsi 1

\section{Introdução}

Anualmente, cerca de 1,1 milhão de mulheres têm diagnóstico de câncer de mama no mundo. Essa incidência representa aproximadamente $10 \%$ de todos os novos casos e $23 \%$ dos casos de câncer em mulheres, sendo este o mais prevalente 1 . Devido a essas estatísticas, o câncer de mama é considerado um grave problema de saúde pública mundial, não só pelo número crescente de casos diagnosticados a cada ano, mas também pelo investimento financeiro que é solicitado para equacionar as questões de diagnóstico e tratamento 2 .

No Brasil, as estimativas para o ano de 2008 apontam que ocorrerão 466.730 casos novos de câncer. O número de casos novos de câncer de mama esperado é de 49.400, com um risco estimado de 50,71 casos a cada 100 mil mulheres. No Sul, desconsiderando os casos de tumores de pele não-melanoma, esse tipo de câncer é o mais freqüente nas mulheres, com um risco estimado de 67,09 casos para cada 100 mil mulheres ${ }^{3}$.

A sobrevida mais favorável encontra-se em países desenvolvidos e a pior sobrevida em alguns países em desenvolvimento, resultado das diferentes taxas de mortalidade no mundo 4 . Os diferentes estadiamentos apresentam prognósticos diferenciados, e estas diferenças são dramáticas. Para pacientes em estádio 0 ou I, somente 1 em 8 mulheres morre em dez anos. Para mulheres com diagnóstico em estádio II, um terço 
morre em dez anos, e para os estágios III e IV o prognóstico é muito pobre, sendo que poucas mulheres de estádio IV permanecem vivas ao fim de dez anos 5 .

Além do estadiamento, outros fatores prognósticos estão relacionados diretamente com a sobrevida das mulheres com câncer de mama, dentre eles, a idade ao diagnóstico, o tipo histológico do tumor e status do receptor hormonal 6 .

As pesquisas epidemiológicas indicam que a incidência e sobrevida de câncer de mama são influenciadas também por fatores sócio-econômicos e demográficos. Mulheres com alto nível sócio-econômico apresentam melhor prognóstico 7. Estudos mostram que a raça/cor é um fator importante na sobrevida; mesmo controlando para fatores tumorais, mulheres de ascendência africana apresentam maior risco de morte 8 .

As estatísticas de sobrevida em câncer estão disponíveis com base em registros de câncer e algumas publicações permitem a comparação entre diferentes lugares. As estatísticas de sobrevida estão normalmente apresentadas como sobrevida relativa, ou a probabilidade de morrer de uma causa relativa ao câncer 4 . Esse é o parâmetro mais utilizado para avaliar resultados na área oncológica, inclusive epidemiológica, em que as taxas de mortalidade em séries históricas são de alta relevância analítica, sendo possível abordar técnicas estatísticas de análise de sobrevida com observações obtidas em registros de serviços de saúde ${ }^{9}$. Além disso, esses estudos permitem ao profissional de saúde conhecer o comportamento da doença, possibilitando uma abordagem técnica realista e que proporcione maior qualidade de vida às pessoas acometidas por esta doença 10 .

No Brasil, poucos são os estudos que analisam a sobrevida geral de pessoas que tiveram diagnóstico de câncer, em especial o câncer de mama. Em Santa Catarina, dois estudos foram encontrados: um analisa a sobrevida em mulheres com mais de 65 anos 11, o outro, a sobrevida livre de doença de mulheres com idade inferior a 35 anos 12 .

Neste estudo, o principal objetivo é definir a sobrevida em cinco anos de mulheres com diagnóstico de câncer de mama, catalogadas nos registros hospitalares de câncer do Centro de Pesquisas Oncológicas de Santa Catarina (CEPON; http://www.fahece.org.br/cepon.htm, acessado em 04/Abr/2008) e do Hospital de Caridade - Irmandade Nosso Senhor dos Passos (http: / / www. hospitaldecaridade.com.br, acessado em 04/ Abr/2008), ambos localizados em Florianópolis, Estado de Santa Catarina, assim como os fatores associados à mesma.

\section{Metodologia}

Este estudo caracteriza-se como de coorte histórica 13 , realizado com dados de mulheres com câncer de mama que tiveram diagnóstico entre 1o de janeiro de 2000 e 31 de dezembro de 2002. Esses dados foram obtidos nos registros hospitalares de câncer do CEPON e do Hospital de Caridade - Irmandade Nosso Senhor dos Passos, ambos de Florianópolis.

Esses dois centros de tratamento do câncer se caracterizam como Centros de Assistência de Alta Complexidade em Oncologia (CACONs), que oferecem assistência especializada e integral aos pacientes com câncer, e deve dispor e manter em funcionamento o registro hospitalar de câncer, fontes sistemáticas de informações dos casos de neoplasia maligna diagnosticados e tratados nos hospitais nos quais se encontram, segundo os critérios estabelecidos pelo Instituto Nacional de Câncer (INCA) 14. Segundo Serrano 15, em Santa Catarina no ano 2000 estavam credenciados pelo INCA seis CACONs, destes, quatro em Florianópolis (Hospital Infantil Joana de Gusmão, Hospital de Caridade - Irmandade Nosso Senhor dos Passos, Hospital Universitário e CEPON), um em Joinville e outro em Blumenau. Desses, quatros hospitais não foram incluídos no presente estudo pois o Hospital Infantil Joana de Gusmão não atende câncer de mama, o Hospital Universitário não possuía registro hospitalar de câncer implantado, e os hospitais de Joinville e Blumenau não responderam aos contatos.

O CEPON é o órgão central do sistema de prevenção e assistência, na área do câncer, em Santa Catarina. Tem como funções o controle e avaliação das atividades e pesquisas oncológicas, de acordo com a política estadual de saúde e normas federais pertinentes. Todas as pessoas são atendidas pelo Sistema Único de Saúde (SUS). O Hospital de Caridade - Irmandade Nosso Senhor dos Passos é administrado pela Irmandade do Senhor Jesus dos Passos, uma entidade de fins religiosos e filantrópicos que promove assistência médico-cirúrgica, hospitalar e ambulatorial. Nesse hospital encontra-se um dos serviços de radioterapia da Grande Florianópolis, e atende pessoas pelo SUS e convênios privados.

As informações referentes aos óbitos, como data e causa básica do óbito, foram coletadas no Sistema de Informação sobre Mortalidade (SIM) do Ministério da Saúde do Estado de Santa Catarina, que é baseado na declaração de óbito. Esse banco de dados foi disponibilizado pela Secretaria de Saúde do Estado de Santa Catarina e foram analisados os bancos dos anos de 2000 a 2007.

A data final do acompanhamento da coorte foi considerada a última data do SIM, dia 31 de 
dezembro de 2007. Foram excluídas do banco do registro hospitalar de câncer as mulheres residentes em outros estados.

O relacionamento entre os bancos de dados do registro hospitalar de câncer e do SIM foi realizado usando-se o programa RecLink II (http:/ / paginas.terra.com.br/educacao/kencamargo/ RecLinkII.html). Esse sistema de relacionamento de bases de dados é fundamentado na técnica de relacionamento probabilístico de registros, na qual o usuário designa as regras de associação entre duas tabelas 16. Foram utilizadas as seguintes variáveis do SIM: número da declaração de óbito, nome, data de nascimento, data de óbito, causa básica da morte e município de residência; e do banco do registro hospitalar de câncer foram usadas as seguintes variáveis: número do prontuário, nome, data de nascimento, município de residência, possibilitando assim o relacionamento dos dados. Após o relacionamento dos bancos de dados foi criado um único banco contendo todas as informações pertinentes ao estudo, permitindo a análise dos dados.

As variáveis do estudo foram faixa etária (abaixo de 30 anos, 30-39 anos, 40-49, 50-59, 6069,70 ou mais anos), estado civil (casada, solteira, divorciada, viúva), escolaridade (nível superior, 2o grau, 1o grau, analfabeto), raça/cores [branca, outras raças/cor agrupadas (preta, parda, indígena e amarela)], diagnóstico e tratamentos anteriores (com diagnóstico/com tratamento; com diagnóstico/sem tratamento, sem diagnóstico/sem tratamento), estádio clínico (EC I, EC II, EC III e EC IV), tipo histológico (carcinoma ductal infiltrante, carcinomas sem infiltração, outros tipos de carcinoma, outros tipos histológicos), tratamentos recebidos (associações com quimioterapia e associações sem quimioterapia). A variável tratamentos recebidos é derivada, no banco original, da variável primeiro tratamento recebido no hospital, que considera os tratamentos realizados no período de quatro meses, a contar da data de início do tratamento 14.

O tempo de sobrevida foi calculado como o intervalo entre a data do diagnóstico (biópsia ou cirurgia) constante no banco do registro hospitalar de câncer e a data do óbito ou final do acompanhamento. O acompanhamento máximo foi de cinco anos, sendo que as mulheres que permaneceram vivas após este período foram censuradas. A data da censura, para as mulheres que não foram a óbito durante o acompanhamento, foi considera como a do final do acompanhamento (31 de dezembro de 2007), pois em Santa Catarina, o SIM tem uma cobertura acima de $90 \% 17$.

As curvas de sobrevida foram estimadas pelo método de Kaplan-Meier, com e sem estratifica- ção. Nesse método a probabilidade de sobrevida até a data especificada é estimada considerandose que a sobrevivência até cada tempo é independente da sobrevivência até outros tempos 18 .

Para comparar as curvas de sobrevida estratificadas foi utilizado o teste log-rank, que compara os valores observados e esperados de cada estrato sob a hipótese nula de que o risco é o mesmo em todos os estratos 18.

A estimação do efeito das co-variáveis foi realizada pelo modelo semiparamétrico de riscos proporcionais, modelo de Cox, que estima a proporcionalidade dos riscos ao longo de todo o tempo de observação ${ }^{18}$. As variáveis incluídas no modelo de Cox foram as que apresentaram o valor de $\mathrm{p}<0,05$, sendo as seguintes: faixa etária, raça, escolaridade, tipo histológico, estádio clínico, diagnóstico e tratamentos anteriores, e tratamentos recebidos. Para a exclusão do modelo foi admitido o valor de $\mathrm{p}>0,10$. Utilizou-se o valor de $\mathrm{p}$ do teste de Wald 18 .

Para avaliação do poder explicativo das covariáveis no tempo de sobrevida foi utilizado o pseudo- $\mathrm{R}^{2}$, medida que estima a proporção da variabilidade total da resposta explicada pelas co-variáveis 18 .

A análise de resíduos de Schoenfelder foi utilizada para avaliar se cada variável respeita a proporcionalidade de riscos do modelo, sendo o risco o mesmo durante todo o tempo de observação. À análise de resíduos é adicionada a correlação linear entre o tempo de sobrevida e o resíduo, neste caso a hipótese nula é de correlação igual a zero, e o valor de $\mathrm{p}>$ 0,05 não rejeita a hipótese nula de proporcionalidade dos riscos 18 .

Para a análise dos dados e construção dos gráficos de sobrevida, utilizou-se o programa estatístico R 2.5.0 (The R Foundation for Statistical Computing, Viena, Áustria; http://www. r-project.org).

\section{Resultados}

No período de janeiro de 2000 a dezembro de 2002, foram catalogadas 1.008 mulheres com o diagnóstico de câncer de mama nos registros hospitalares de câncer, sendo excluídas seis por serem residentes em outros estados, totalizando 1.002 residentes em Santa Catarina. No período de acompanhamento desta coorte, cinco anos, ocorreram 262 óbitos, sendo 235 por câncer de mama e 27 por outras causas, as quais foram censuradas.

Dessas mulheres catalogadas nos registros hospitalares de câncer, $50 \%$ eram provenientes da Grande Florianópolis, 24\% do sul catarinense e as demais do restante do estado. 
A sobrevida geral observada nesta coorte aos 12 meses foi de $95,7 \%$ (intervalo de $95 \%$ de confiança - IC95\%: 94,4-97,0), aos 24 meses foi de 88,3\% (IC95\%: 86,3-90,3), aos 36 meses, 83,4\% (IC95\%: 81,2-85,8), aos 48 meses, 79,4\% (IC95\%: $76,9-81,9)$ e aos 60 meses, 76,2\% (IC95\%: 73,678,9) (Figura 1a).

A idade média observada entre as mulheres foi de 53,85 anos (desvio-padrão - DP $=13,06$ anos), sendo a mediana de 52 anos (1o quartil: 45 anos, 3o quartil: 63 anos; idade mínima de 13 anos e máxima de 89). A sobrevida em relação à idade mostrou que mulheres com idade inferior a 30 anos têm uma sobrevida pior que as das demais faixas etárias, sendo que aos 57 meses, somente $46,7 \%$ delas permaneciam vivas (Figura 1b). A faixa etária acima dos 70 anos apresentou $14,4 \%$ dos diagnósticos, com uma taxa de sobrevida de $70,5 \%$, sendo a segunda pior taxa de sobrevida. A faixa etária de 40-49 anos foi a com a melhor taxa de sobrevida, com $82,1 \%$, sendo que representaram $27,5 \%$ dos diagnósticos realizados. A faixa etária de 50-59 anos representou $28,8 \%$ dos casos, com uma sobrevida de $75,5 \%$. A faixa etária de 60-69 anos correspondeu a 16,6\% dos diagnósticos, com uma taxa de sobrevida de $74,3 \%$ (Tabela 1). Mulheres entre 30-39 anos representaram $11,3 \%$ dos casos, com um taxa de sobrevida de $77 \%$ (Tabela 1 ).

Figura 1

Curvas de sobrevida em cinco anos de mulheres com câncer de mama. Santa Catarina, Brasil, 2000-2002.

1a) Sobrevida geral

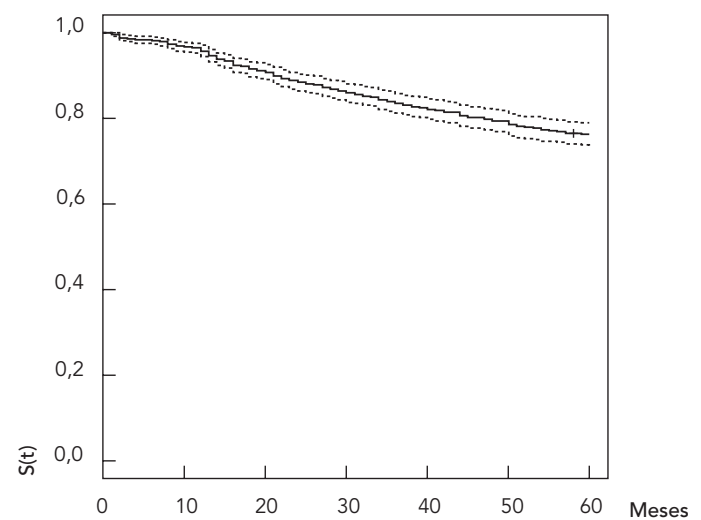

1c) Estratificada por raça/cor

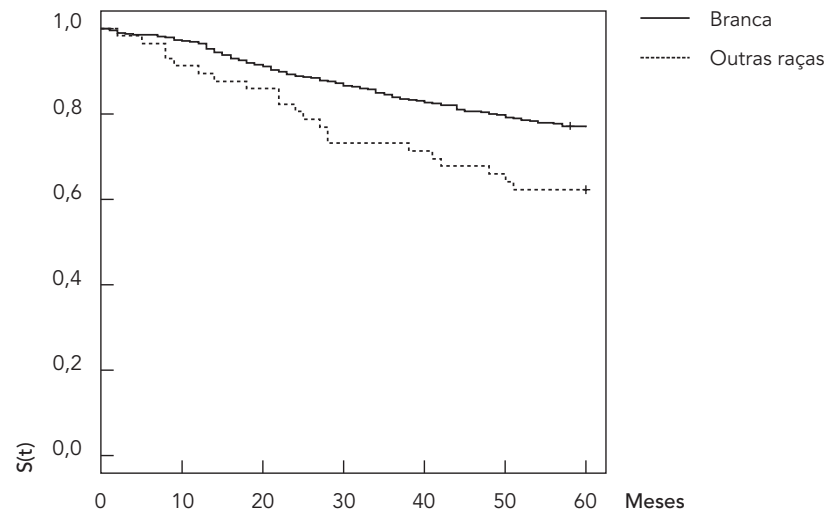

1b) Estratificada por faixa etária (anos)

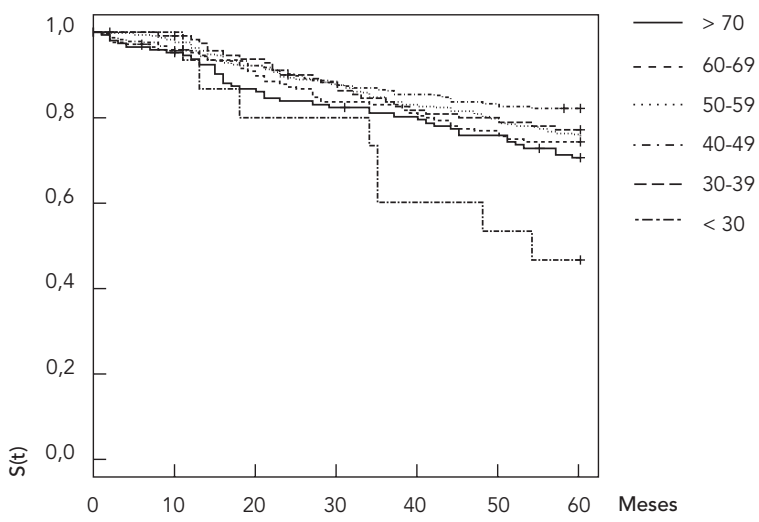

1d) Estratificada por escolaridade

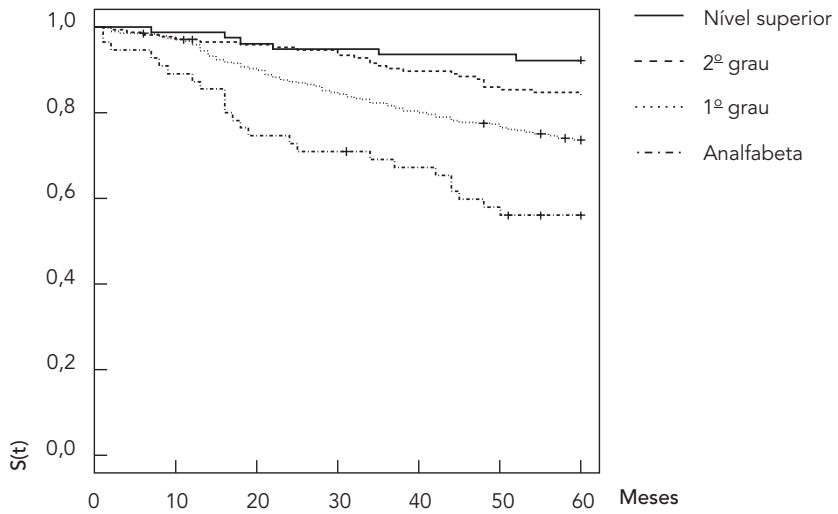

(continua) 
1e) Estratificada por estado civil

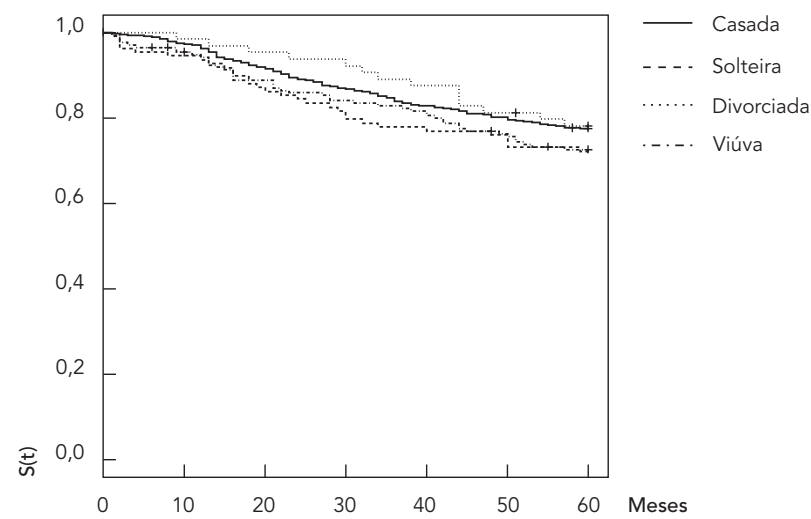

1g) Estratificada por histologia tumoral

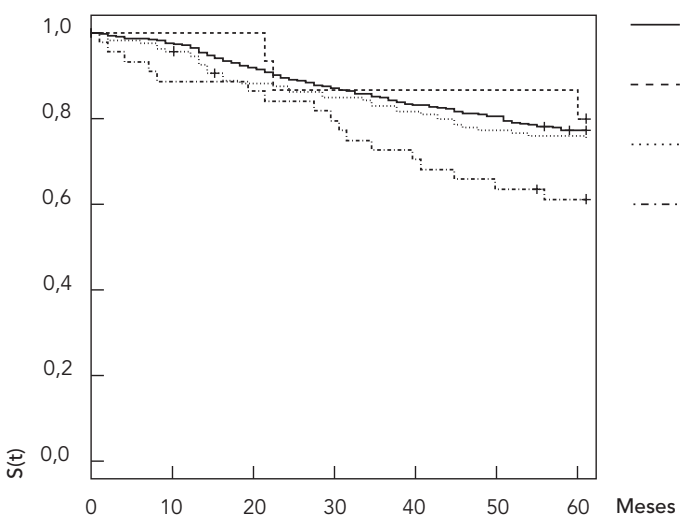

1f) Estratificada por estádio clínico

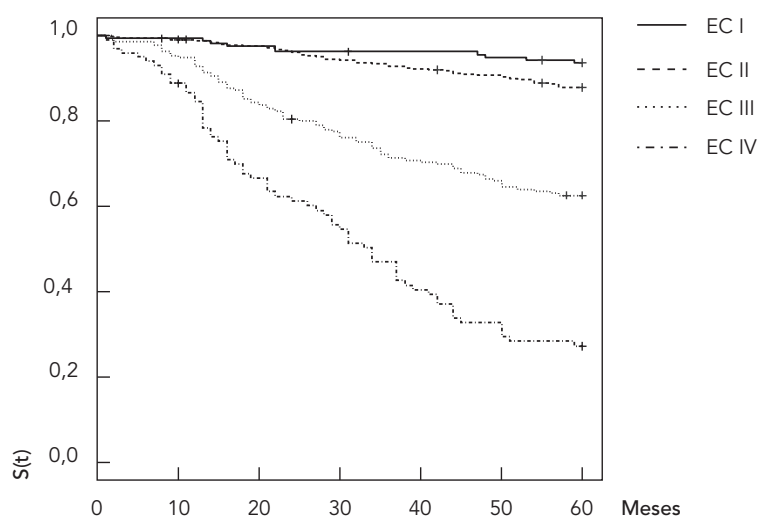

1h) Estratificada por diagnóstico e tratamento anterior

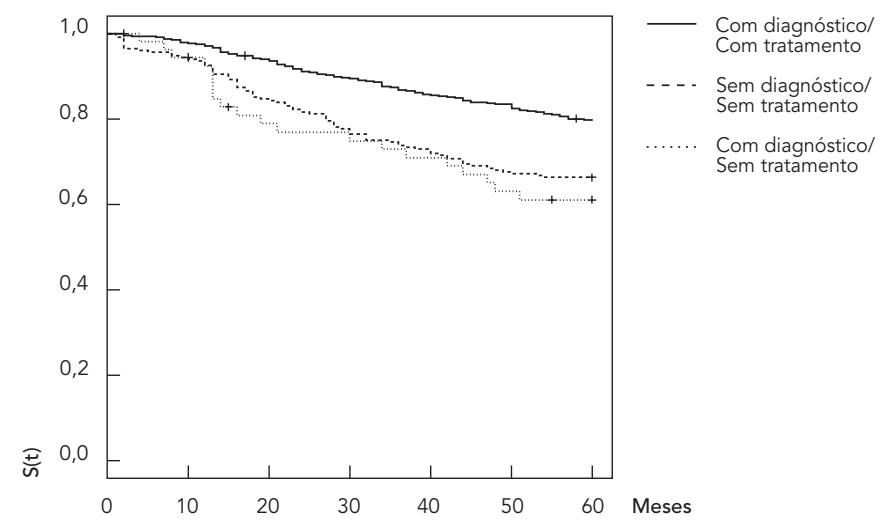

1i) Estratificada por tratamento

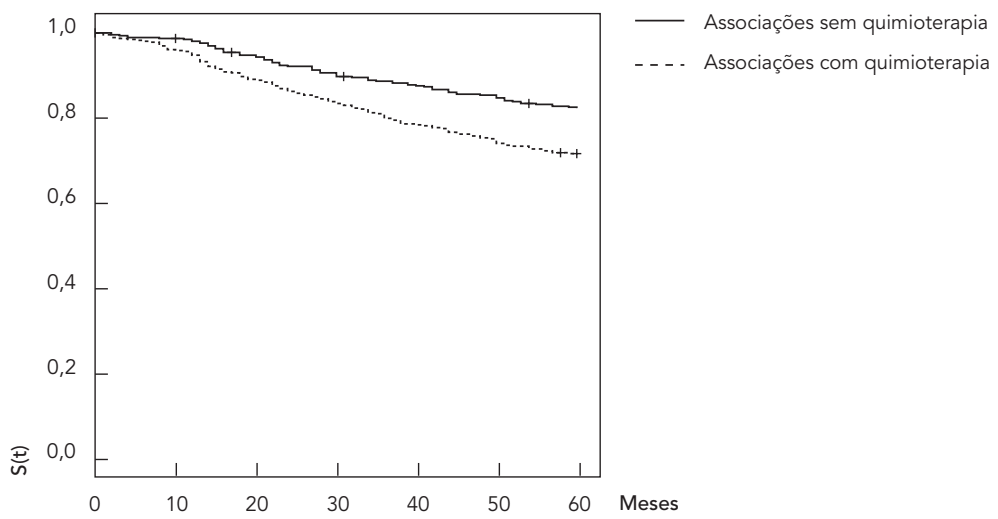


Tabela 1

Análise univariada, sobrevida e razão de risco em mulheres com câncer de mama. Florianópolis, Santa Catarina, Brasil, $2000-2002$.

\begin{tabular}{|c|c|c|c|c|c|c|}
\hline Variáveis & n (\%) & Óbitos (\%) & $\begin{array}{c}\text { Sobrevida } \\
60 \text { meses (\%) }\end{array}$ & $\begin{array}{c}\text { Teste log-rank } \\
\text { (valor de p) }\end{array}$ & $\begin{array}{l}\text { Hazard ratio } \\
\text { (IC95\%) }\end{array}$ & $\begin{array}{l}\text { Teste Wald } \\
\text { (valor de p) }\end{array}$ \\
\hline \multicolumn{7}{|l|}{ Faixa etária (anos) } \\
\hline Acima 70 & $144(14,4)$ & $41(28,5)$ & $70,5(63,3-78,5)$ & 0,008 & 1,00 & 0,012 \\
\hline $60-69$ & $166(16,6)$ & $42(25,3)$ & $74,3(67,9-81,3)$ & & $0,85(0,55-1,31)$ & \\
\hline $50-59$ & $288(28,8)$ & $70(24,3)$ & $75,5(70,7-80,7)$ & & $0,79(0,54-1,16)$ & \\
\hline $40-49$ & $275(27,5)$ & $49(17,8)$ & $82,1(77,7-86,8)$ & & $0,57(0,38-0,87)$ & \\
\hline $30-39$ & $113(11,3)$ & $25(22,1)$ & $77,0(69,5-85,3)$ & & $0,74(0,45-1,21)$ & \\
\hline Abaixo 30 & $15(1,5)$ & $8(53,3)$ & $46,7(27,2-80,2)$ & & $2,02(0,95-4,32)$ & \\
\hline Total & $1.001(100,0)$ & $235(23,5)$ & & & & \\
\hline \multicolumn{7}{|l|}{ Raça/cor } \\
\hline Branca & $915(94,1)$ & $208(22,7)$ & $76,9(74,2-79,7)$ & 0,007 & 1,00 & 0,008 \\
\hline Outras raças/cores & $57(5,9)$ & $21(36,8)$ & $62,2(50,7-76,4)$ & & $1,84(1,17-2,88)$ & \\
\hline Total & $972(100,0)$ & $229(23,6)$ & & & & \\
\hline \multicolumn{7}{|l|}{ Estado civil } \\
\hline Casada & $644(64,9)$ & $143(22,2)$ & $77,6(74,4-80,9)$ & 0,283 & 1,00 & 0,285 \\
\hline Solteira & $110(11,1)$ & $31(28,2)$ & $71,3(63,2-80,4)$ & & $1,35(0,92-1,99)$ & \\
\hline Divorciada & $64(6,5)$ & $14(21,9)$ & $78,1(68,5-88,9)$ & & $0,94(0,55-1,63)$ & \\
\hline Viúva & $174(17,5)$ & $46(26,4)$ & $72,6(66,2-79,7)$ & & $1,26(0,91-1,76)$ & \\
\hline Total & $992(100,0)$ & $234(23,6)$ & & & & \\
\hline \multicolumn{7}{|l|}{ Escolaridade } \\
\hline Nível superior & $77(8,9)$ & $6(7,8)$ & $92,2(86,4-98,4)$ & 0,000 & 1,00 & 0,000 \\
\hline 2o grau & $165(19,1)$ & $26(15,8)$ & $84,0(78,6-89,9)$ & & $2,11(0,87-5,13)$ & \\
\hline 1ㅇ grau & $567(65,6)$ & $147(25,9)$ & $73,6(70,0-77,3)$ & & $3,76(1,66-8,50)$ & \\
\hline Analfabeta & $55(6,4)$ & $24(43,6)$ & $56,0(44,2-70,9)$ & & $7,40(3,02-18,1)$ & \\
\hline Total & $864(100,0)$ & $203(23,5)$ & & & & \\
\hline \multicolumn{7}{|l|}{ Estádio clínico } \\
\hline $\mathrm{ECl}$ & $156(18,1)$ & $10(6,4)$ & $93,6(89,8-97,5)$ & 0,000 & 1,00 & 0,000 \\
\hline EC II & $398(46,2)$ & $48(12,1)$ & $87,8(84,6-91,1)$ & & $1,94(0,98-3,84)$ & \\
\hline EC III & $209(24,3)$ & $78(37,3)$ & $62,5(56,3-69,5)$ & & $7,18(3,72-13,88)$ & \\
\hline EC IV & $98(11,4)$ & $68(69,4)$ & $27,3(19,6-38,1)$ & & $19,49(10,02-37,94)$ & \\
\hline Total & $861(100,0)$ & $204(23,7)$ & & & & \\
\hline \multicolumn{7}{|l|}{ Histologia } \\
\hline Carcinoma ductal infiltrante & $777(77,7)$ & $175(22,5)$ & $77,2(74,3-80,3)$ & 0,076 & 1,00 & 0,083 \\
\hline Carcinoma sem infiltração & $15(1,5)$ & $3(13,6)$ & $80,0(62,1-1,00)$ & & $0,85(0,27-2,65)$ & \\
\hline Demais tipos de carcinoma & $163(16,3)$ & $39(24,2)$ & $75,3(68,9-82,4)$ & & $1,12(0,79-1,58)$ & \\
\hline Outros tipos histológicos & $45(4,5)$ & $17(42,5)$ & $61,3(48,4-77,5)$ & & $1,90(1,16-3,13)$ & \\
\hline Total & $1.000(100,0)$ & $234(23,4)$ & & & & \\
\hline \multicolumn{7}{|l|}{ Diagnóstico e tratamentos anteriores } \\
\hline Com diagnóstico/com tratamento & $660(69,8)$ & $133(20,2)$ & $79,6(76,5-82,7)$ & 0,000 & 1,00 & 0,000 \\
\hline Com diagnóstico/sem tratamento & $231(24,4)$ & $77(33,3)$ & $66,3(60,5-72,7)$ & & $1,86(1,41-2,47)$ & \\
\hline Sem diagnóstico/sem tratamento & $55(5,8)$ & $20(36,4)$ & $61,1(49,1-76,0)$ & & $2,24(1,40-3,58)$ & \\
\hline Total & $946(100,0)$ & $230(24,3)$ & & & & \\
\hline \multicolumn{7}{|l|}{ Tratamentos recebidos } \\
\hline Associações sem quimioterapia & $435(46,2)$ & $76(17,5)$ & $82,2(78,7-85,9)$ & 0,000 & 1,00 & 0,000 \\
\hline Associações com quimioterapia & $507(53,8)$ & $142(53,8)$ & $71,7(67,8-75,7)$ & & $1,72(1,30-2,27)$ & \\
\hline Total & $942(100,0)$ & $218(23,1)$ & & & & \\
\hline
\end{tabular}


As variáveis sócio-econômicas raça/cor e escolaridade mostraram-se significativas em relação à sobrevida. A raça/cor branca foi predominante e apresentou melhor sobrevida $(76,9 \%)$ que as raças/cores negra, parda, amarela e indígena agrupadas (62,2\%) (log-rank, p = 0,007) (Figura 1c), a razão de risco da categoria de raças/cores agrupadas foi $84 \%$ maior que a raça/cor branca (Tabela 1).

A sobrevida estratificada por escolaridade mostrou que mulheres com nível superior apresentam melhor sobrevida global em cinco anos $(92,2 \%)$ quando comparadas às mulheres com 2 o grau (84\%), ำ grau (73,6\%) e analfabetas (56\%) (log-rank, p =0,000) (Figura 1d), sendo que as mulheres analfabetas tiveram um risco 7,40 vezes maior de morrer que as de nível superior, e as com 1o grau, risco 3,76 vezes maior (Tabela 1 ).

O estado civil não mostrou significância em relação à sobrevida, mas percebe-se que as mulheres solteiras tiveram pior sobrevida (Figura 1e).

O estádio clínico mostrou-se importante fator associado à sobrevida. Somente $18,1 \%$ das mulheres tiveram diagnóstico em EC I (Tabela 1), e estas tiveram a melhor sobrevida global aos cinco anos (93,6\%), enquanto as mulheres com diagnóstico em EC IV tiveram a pior sobrevida ( $p=0,000$ ), com a mediana de 34 meses (IC95\%: 27-42 meses) e sobrevida global aos cinco anos de $27,3 \%$ (Figura 1f). O risco de óbito das mulheres com EC III foi 7,18 vezes maior que as em EC I, e as com EC IV no diagnóstico foi de 19,49 vezes maior (Tabela 1).

O tipo histológico mais freqüente foi o carcinoma ductal infiltrante $(77,7 \%)$ (Tabela 1$)$. A melhor taxa de sobrevida foi observada entre as mulheres que tiveram carcinoma sem infiltração ao diagnóstico (80\%). Os outros tipos histológicos, diferentes de carcinomas, foram os que mostraram maior risco de óbito, 2,22 vezes maior que as com diagnóstico de carcinoma ductal infiltrante (Tabela 1, Figura 1g).

Mulheres que chegaram aos hospitais de referência com diagnóstico e tratamento anteriores tiveram melhor sobrevida que as demais (Figura 1h). As que chegaram aos registros hospitalares de câncer sem diagnóstico e sem tratamento tiveram um risco 2,24 vezes maior de óbito que as mulheres com diagnóstico e tratamento anteriores à admissão (Tabela 1).

Em relação aos tratamentos recebidos, as mulheres que receberam como tratamento associações sem quimioterapia tiveram melhor sobrevida (82,2\%) quando comparadas às associações de tratamento com quimioterapia $(71,7 \%)$ (Tabela 1, Figura 1i).
Na análise de Cox foram incluídas as variáveis que apresentaram efeito significativo em relação à sobrevida, as quais foram faixa etária, raça/cor, escolaridade, tipo histológico, estádio clínico, diagnóstico e tratamentos anteriores e tratamentos recebidos. As variáveis foram incluídas uma a uma, conforme apresentado na Tabela 2.

A faixa etária e raça/cor foram as primeiras variáveis a serem incluídas (modelo 1), seguidas da escolaridade (modelo 2). O tipo histológico foi adicionado à análise no modelo 3 , o qual não apresentou significância estatística. Em seguida, foi incluída a variável estádio clínico (modelo 4), e a variável raça/cor perdeu sua significância. Por fim, foram incluídas as variáveis diagnóstico e tratamentos anteriores (modelo 5), e tratamentos recebidos (modelo 6). No modelo 6, a variável diagnóstico e tratamentos anteriores perdeu sua significância, e a variável tratamento recebido não mostrou-se significante, sendo realizada a análise final (modelo 7) com as variáveis faixa etária, escolaridade e estádio clínico. Esse modelo foi assumido como o modelo final na análise de Cox.

No modelo 2, com a inclusão da variável escolaridade, percebe-se um aumento do risco de óbito das mulheres com idade inferior a 30 anos, que passa, no modelo 1, de hazard ratio $-\mathrm{HR}=$ 2,05 (IC95\%: 0,96-4,39) para HR = 3,43 (IC95\%: 1,55-7,59). No modelo 4 , com a inclusão do estadiamento, percebe-se que o risco de óbito das mulheres analfabetas diminui consideravelmente, de HR = 6,91 (IC95\%: 2,71-17,62), no modelo 3, para HR = 3,71 (IC95\%: 1,44-9,55) (Tabela 2).

$\mathrm{Na}$ análise ajustada (Tabela 2) percebeu-se que: a faixa etária com maior risco de óbito foi a menor de 30 anos, com um risco 4,15 vezes maior que as mulheres com mais de 70 anos; a escolaridade, as mulheres analfabetas apresentaram um risco de óbito 3,90 vezes maior que as com nível superior; o EC IV apresentou um risco de óbito 16,47 vezes maior que EC I, enquanto o EC III mostrou um risco 6,55 vezes maior de óbito.

$\mathrm{O}$ ajuste do modelo final foi dado pelo valor do pseudo- $R^{2}$, igual a 0,245 . A análise de resíduos de Schoenfelder foi realizada com o modelo final, sendo aceita a hipótese nula de igualdade de riscos ao longo do tempo, comprovando a proporcionalidade do risco de óbito, e que, portanto, as variáveis não são tempos dependentes $(\mathrm{p}=0,24)$.

\section{Discussão}

Este estudo permitiu conhecer características das mulheres com câncer de mama atendidas nos dois serviços de Florianópolis, CEPON e Hospital de Caridade - Irmandade Nosso Senhor dos 
Análise multivariada em mulheres com câncer de mama. Florianópolis, Santa Catarina, Brasil, 2000-2002.

\begin{tabular}{|c|c|c|c|c|c|c|c|}
\hline \multirow[t]{2}{*}{ Variáveis } & \multicolumn{7}{|c|}{ Hazard ratio (IC95\%) } \\
\hline & $\begin{array}{l}\text { Modelo } 1 \\
(n=971)\end{array}$ & $\begin{array}{c}\text { Modelo } 2 \\
(n=840)\end{array}$ & $\begin{array}{l}\text { Modelo } 3 \\
(n=839)\end{array}$ & $\begin{array}{c}\text { Modelo } 4 \\
(n=736)\end{array}$ & $\begin{array}{l}\text { Modelo } 5 \\
(\mathrm{n}=715)\end{array}$ & $\begin{array}{l}\text { Modelo } 6 \\
(\mathrm{n}=697)\end{array}$ & $\begin{array}{l}\text { Modelo } 7 \\
(n=755)\end{array}$ \\
\hline \multicolumn{8}{|l|}{ Faixa etária (anos) } \\
\hline Acima 70 & 1,00 & 1,00 & 1,00 & 1,00 & 1,00 & 1,00 & 1,00 \\
\hline \multirow[t]{2}{*}{$60-69$} & 0,83 & 0,89 & 0,91 & 0,85 & 0,87 & 0,87 & 0,90 \\
\hline & $(0,54-1,29)$ & $(0,54-1,46)$ & $(0,56-1,50)$ & $(0,50-1,44)$ & $(0,51-1,47)$ & $(0,51-1,52)$ & $(0,54-1,52)$ \\
\hline \multirow[t]{2}{*}{$50-59$} & 0,81 & 1,06 & 1,10 & 1,14 & 0,94 & 0,86 & 1,05 \\
\hline & $(0,55-1,19)$ & $(0,69-1,65)$ & $(0,70-1,71)$ & $(0,72-1,81)$ & $(0,59-1,51)$ & $(0,51-1,43)$ & $(0,67-1,67)$ \\
\hline \multirow[t]{2}{*}{$40-49$} & 0,57 & 0,76 & 0,78 & 0,85 & 0,81 & 0,69 & 0,84 \\
\hline & $(0,31-0,86)$ & $(0,47-1,22)$ & $(0,49-1,26)$ & $(0,52-1,38)$ & $(0,49-1,32)$ & $(0,40-1,19)$ & $(0,51-1,36)$ \\
\hline \multirow[t]{2}{*}{$30-39$} & 0,71 & 0,96 & 1,00 & 1,06 & 1,03 & 0,92 & 1,08 \\
\hline & $(0,42-1,17)$ & $(0,55-1,70)$ & $(0,56-1,77)$ & $(0,58-1,94)$ & $(0,57-1,87)$ & $(0,49-1,75)$ & $(0,60-1,95)$ \\
\hline \multirow[t]{2}{*}{ Abaixo 30} & 2,05 & 3,43 & 3,32 & 3,91 & 4,10 & 4,61 & 4,15 \\
\hline & $(0,96-4,39)$ & $(1,55-7,59)$ & $(1,48-7,45)$ & $(1,65-9,29)$ & $(1,74-9,72)$ & $(1,86-11,44)$ & $(1,75-9,81)$ \\
\hline \multicolumn{8}{|l|}{ Raça/cor } \\
\hline Branca & 1,00 & 1,00 & 1,00 & 1,00 & & & \\
\hline \multirow[t]{2}{*}{ Outras raças/cores } & 1,94 & 1,88 & 1,90 & 1,43 & & & \\
\hline & $(1,24-3,06)$ & $(1,17-3,01)$ & $(1,18-3,04)$ & $(0,84-2,44)$ & & & \\
\hline \multicolumn{8}{|l|}{ Escolaridade } \\
\hline Nível superior & & 1,00 & 1,00 & 1,00 & 1,00 & 1,00 & 1,00 \\
\hline \multirow[t]{2}{*}{ 2o grau } & & 2,07 & 2,05 & 1,37 & 1,63 & 1,55 & 1,41 \\
\hline & & $(0,85-5,04)$ & $(0,84-4,99)$ & $(0,55-3,41)$ & $(0,62-4,34)$ & $(0,58-4,12)$ & $(0,57-3,48)$ \\
\hline \multirow[t]{2}{*}{ 1o grau } & & 3,60 & 3,58 & 2,17 & 2,60 & 2,58 & 2,24 \\
\hline & & $(1,58-8,21)$ & $(1,57-8,15)$ & $(0,93-5,05)$ & $(1,03-6,53)$ & $(1,01-6,56)$ & $(0,96-5,23)$ \\
\hline \multirow[t]{2}{*}{ Analfabeta } & & 7,31 & 6,91 & 3,71 & 3,72 & 3,81 & 3,90 \\
\hline & & $(2,89-18,5)$ & $(2,71-17,62)$ & $(1,44-9,55)$ & $(1,33-6,53)$ & $(1,34-10,83)$ & $(1,52-10,00)$ \\
\hline \multicolumn{8}{|l|}{ Histologia } \\
\hline \multicolumn{2}{|l|}{ Carcinoma ductal infiltrante } & & 1,00 & & & & \\
\hline \multirow[t]{2}{*}{ Carcinoma sem infiltração } & & & 0,91 & & & & \\
\hline & & & $(0,29-2,89)$ & & & & \\
\hline \multirow[t]{2}{*}{ Outros tipos de carcinoma } & & & 1,02 & & & & \\
\hline & & & $(0,70-1,49)$ & & & & \\
\hline Demais tipos histológicos & & & 1,36 & & & & \\
\hline & & & $(0,65-2,84)$ & & & & \\
\hline Estádio clínico & & & & & & & \\
\hline EC I & & & & 1,00 & 1,00 & 1,00 & 1,00 \\
\hline EC II & & & & 1,66 & 1,58 & 1,40 & 1,67 \\
\hline & & & & $(0,80-3,43)$ & $(0,46-3,27)$ & $(0,67-2,94)$ & $(0,80-3,46)$ \\
\hline EC III & & & & 6,37 & 5,81 & 4,80 & 6,55 \\
\hline & & & & $(3,15-12,87)$ & $(2,87-11,73)$ & $(2,32-9,91)$ & $(3,24-13,21)$ \\
\hline EC IV & & & & 17,61 & 14,44 & 12,41 & 16,47 \\
\hline & & & & $(8,62-35,97)$ & $(7,08-29,46)$ & $(6,01-25-62)$ & $(9,08-33,56)$ \\
\hline Diagnóstico e tratamentos anteriores & & & & & & & \\
\hline Com diagnóstico/com tratamento & & & & & 1,00 & 1,00 & \\
\hline Com diagnóstico/sem tratamento & & & & & 1,39 & 1,38 & \\
\hline & & & & & & $(1,00-1,94)$ & $(0,98-1,95)$ \\
\hline Sem diagnóstico/sem tratamento & & & & & 1,63 & 1,56 & \\
\hline & & & & & & $(0,94-2,82)$ & $(0,89-2,76)$ \\
\hline Tratamentos recebidos & & & & & & & \\
\hline Associações com quimioterapia & & & & & & 1,00 & \\
\hline Associações sem quimioterapia & & & & & & 1,37 & \\
\hline & & & & & & $(0,93-2,02)$ & \\
\hline Teste de Wald (valor de p) & 0,001 & 0,000 & 0,000 & 0,000 & 0,000 & 0,000 & 0,000 \\
\hline Pseudo- $\mathrm{R}^{2}$ & 0,020 & 0,056 & 0,055 & 0,252 & 0,255 & 0,255 & 0,245 \\
\hline
\end{tabular}


Passos, sendo possível definir a taxa de sobrevida geral após cinco anos do diagnóstico, 76,2\%, e os fatores associados à sobrevida: faixa etária, escolaridade e estadiamento.

$\mathrm{O}$ estudo foi realizado com base em dados secundários, estando sujeito a limitações, que podem, em maior ou menor grau, interferir nos resultados apresentados. Dentre essas, pode-se citar a falta de padronização no preenchimento das fichas; dados registrados por diferentes profissionais, podendo gerar erros de digitação, apesar de serem coletados e digitados por registradoras treinadas e supervisionadas por uma epidemiologista, treinada pelo Programa de Avaliação e Vigilância do Câncer e seus fatores de risco (PAV). Também podem ter ocorrido problemas na definição das variáveis, como a variável tratamentos recebidos, que deve ser preenchida somente com os tratamentos recebidos nos quatro meses anteriores à entrada no hospital. Os resultados encontrados não podem ser utilizados como parâmetros para toda a população de Santa Catarina, pois, mesmo contando com dados de pessoas de todo o estado, as análises foram realizadas somente com dados de dois serviços com registro hospitalar de câncer do estado.

Entre as variáveis relacionadas com o risco de morte por câncer de mama, na literatura, encontram-se as sócio-econômicas, métodos de diagnóstico, estadiamento, tipo histológico, diferenciação, status dos receptores hormonais, cirurgia, radioterapia, hormonioterapia e quimioterapia 19.

A taxa de sobrevida geral após cinco anos neste estudo foi de $76,2 \%$. Ao comparar essa taxa com as de outros estudos brasileiros, percebe-se que somente em Santa Maria (Rio Grande do Sul) a taxa de sobrevida geral foi maior, de $87,7 \% 9$. Em mulheres atendidas no INCA (Rio de Janeiro) essa taxa foi de $75 \%$ 20; em Goiânia (Goiás), entre mulheres catalogadas pelo registro de câncer de base populacional foi $57 \% 21$, nos casos registrados no registro de câncer de base populacional de Belém (Pará) esta taxa foi de $61 \%$ 22. Em Florianópolis, em um serviço de referência de mastologia, em mulheres com mais de 65 anos, a taxa de sobrevida geral em cinco anos foi de $73,1 \% 11$.

A idade mostrou-se como um fator independente na sobrevida nesta coorte. O risco de óbito foi maior em mulheres jovens, abaixo de 30 anos, possivelmente devido ao câncer de mama ser mais agressivo e ter pior prognóstico nesta faixa etária 19. Segundo a American Cancer Society 23, de 2000 a 2004, 95\% dos casos de câncer diagnosticados e $97 \%$ dos casos de óbito por câncer de mama foram em mulheres com 40 anos ou mais. Neste estudo, encontramos mais de $10 \%$ dos casos em mulheres com menos de 40 anos.
A relação entre nível sócio-econômico e prognóstico da doença é permeada pelo diagnóstico realizado numa fase já avançada da doença, devido à dificuldade de acesso aos programas de prevenção e aos cuidados médicos nas classes sociais menos favorecidas, mesmo em países desenvolvidos 24 .

A raça/cor mostrou diferente sobrevida entre os dois grupos analisados. Alguns fatores contribuem para explicar as disparidades raciais, como diferença no acesso a serviços de boa qualidade e resolutividade, tempo prolongado até o diagnóstico e tratamentos 24,25. Essas diferenças de atendimento acabam refletindo em mudanças no estadiamento entre os grupos 26 . Além disso, a alta taxa de incidência entre mulheres brancas reflete a combinação de fatores que afetam o diagnóstico e aqueles que afetam o risco da doença (primeira gestação tardia e terapia de reposição hormonal), demonstrando que há uma elevada taxa de incidência em mulheres de raça/ cor branca, e um prognóstico pior naquelas de raça/cor negra 25.

O estado civil não apresentou significância, mas foi perceptível que mulheres solteiras tiveram pior sobrevida. Outro estudo encontrou que mulheres não-casadas, acima de 65 anos, têm aumento do risco de morte por câncer de mama depois de controlados os fatores de risco relevantes, incluindo estadiamento ao diagnóstico e tratamento. É comum mulheres não-casadas serem diagnosticadas com a doença mais avançada 27 .

Outra variável sócio-econômica, a escolaridade, mostrou-se como fator independente na sobrevida. Isso pode ter acontecido devido ao fato de mulheres com maior escolaridade serem mais expostas ao exame clínico das mamas e apresentarem maior freqüência de mamografia, como demonstra um estudo realizado em Botucatu (São Paulo), que analisou as oportunidades de diagnóstico precoce para o câncer de mama na cidade 2 .

O tipo histológico mostrou-se como outro fator importante para a sobrevida, apesar de não ser compreendido como um fator prognóstico. O carcinoma ductal não infiltrante é usualmente diagnosticado por mamografia e a grande incidência está diretamente ligada à habilidade de detectar câncer que não pode ser diagnosticado por exame clínico ou auto-exame 28. A história natural do carcinoma sem invasão é pobremente entendida porque mulheres diagnosticadas nesta fase passam por cirurgia, associada ou não à radioterapia e hormonioterapia.

O estadiamento clínico ao diagnóstico, que reúne o tamanho tumoral, o comprometimento linfonodal e a presença de metástases à distância, mostrou-se como importante fator prognós- 
tico na sobrevida. A sobrevida quando ajustada por tamanho tumoral e comprometimento linfonodal mostra que mulheres com diagnóstico precoce apresentam uma redução do risco de morte 5 .

Para o tratamento do câncer de mama são levados em consideração o tamanho do tumor, estadiamento e outras características, bem como preferências da paciente. O tratamento envolve cirurgia, radioterapia, quimioterapia (antes ou depois da cirurgia), hormonioterapia e agentes biológicos para casos específicos 23 .

Os tratamentos com associação de quimioterapia mostraram maior risco de óbito em comparação às associações sem quimioterapia, contrariando dados segundo os quais a adição de quimioterapia ao tratamento promoveria aumento da sobrevida geral ${ }^{6}$. As limitações dos bancos de dados secundários, como erros de classificação da variável primeiro tratamento recebido no hospital, podem contribuir para explicar o aumento do risco de óbito com a inclusão dos tratamentos de quimioterapia e radioterapia. Outra explicação possível seria o inadequado uso das intervenções terapêuticas, em desacordo com os protocolos clínicos recomendados. Isso foi verificado em estudo de mulheres com câncer de mama no Rio de Janeiro, que mostrou que algumas intervenções recomendadas e com eficácia documentada são subutilizadas, e outras que são desaconselhadas em alguns casos, acabaram sendo realizadas 29 .

No Brasil, com o passar dos anos, percebe-se uma pequena redução nos casos de diagnóstico nos estádios avançados (EC III e IV), passando de 50,6\% dos casos em 1995 para 45,3\% em 200230. Mas não é observada diminuição da mortalidade por câncer de mama nos últimos anos, o que pode ser atribuído, principalmente, a retardamento no diagnóstico e no início de terapêutica adequada 31. Como houve uma pequena melhora no estadiamento no momento do diagnóstico, diminuindo os casos em estádio avançado, é pertinente refletir sobre as intervenções terapêuticas, se as mesmas estão sendo oferecidas de maneira apropriada, pois não estão sendo capazes de interferir no padrão de mortalidade 30 .

Segundo o Consenso do Controle do Câncer de Mama 31, as campanhas de diagnóstico por meio de rastreamento por mamografia devem ser realizadas em mulheres com idade entre 50 e 69 anos, e para mulheres com idade a partir dos 40 anos, o rastreamento deve ser realizado por exame clínico das mamas. Os dados desta coorte mostram que $27,5 \%$ dos casos foram diag- nosticados em mulheres de 40-49 anos, que não são contempladas pela política de rastreamento mamográfico, sendo que a incidência nesta faixa etária é muito superior à da faixa de 60-69 anos, que é contemplada pela política.

A American Cancer Society 23 recomenda o início da mamografia em mulheres acima de 40 anos a cada dois anos, e a partir dos 50 anos, anualmente. Os progressos na redução da mortalidade por câncer de mama podem ser acelerados pelo aumento do número de mulheres rastreadas a partir dos 40 anos de idade. Achados mostram que o rastreamento mamográfico em mulheres com idade entre 40-64 anos é um fator de proteção para mortalidade por câncer de mama 32 . Na Suécia, a redução da mortalidade chegou a $40 \%$ depois da implantação do rastreamento mamográfico ${ }^{33}$. Além da redução da mortalidade, é observado que o diagnóstico em campanhas de rastreamento mamográfico apresenta diminuição dos casos em estádio avançado, comparados a casos diagnosticados por outros métodos 34 .

No entanto, obstáculos à melhoria dos cuidados às pessoas com câncer surgem de várias formas, incluindo déficits em conhecimentos públicos e sensibilização, barreiras sociais e culturais, problemas na organização dos cuidados de saúde, e insuficiência de recursos 35 .

O desenvolvimento de cuidados para a saúde mamária nas regiões com recursos financeiros limitados é um passo crucial para melhoria da saúde 35 . Uma análise dos custos de tratamento mostrou que o tratamento de duas a quatro mulheres com câncer de mama avançado (apenas custos econômicos) pode ser equivalente ao custo do rastreamento de mil mulheres 36 .

Neste estudo, percebe-se que em um estado com um dos maiores índices de desenvolvimento humano (IDH) do país, os fatores sociais ainda mostram-se independentes no estudo da sobrevida de mulheres com câncer de mama. Esses fatores influenciam a sobrevida dessas mulheres independente do estadiamento, variável que pode ser alterada por meio de campanhas de diagnóstico precoce e acesso aos exames de rastreamento, que são de extrema importância para o prognóstico.

Outra questão fundamental são os tratamentos oferecidos a essas mulheres, que podem acarretar um aumento do risco de óbito com a adição dos mesmos. Isso implica pensar se os protocolos disponíveis para a utilização nesses serviços são os mais indicados para os casos apresentados, necessitando de estudos mais aprofundados e minuciosos a este respeito. 


\section{Resumo}

Este estudo tem o objetivo de descrever a sobrevida em câncer de mama e os fatores associados à mesma. Caracteriza-se como uma coorte histórica de mulheres com diagnóstico de câncer de mama no período de 2000 a 2002, que foram catalogadas nos registros hospitalares de câncer do Centro de Pesquisas Oncológicas de Santa Catarina e Hospital de Caridade - Irmandade Nosso Senhor dos Passos, os dois localizados em Florianópolis, Santa Catarina, Brasil. Para a análise estatística foram utilizados o estimador de Kaplan-Meier e o modelo de Cox. A taxa de sobrevida geral em cinco anos foi de 76,2\% (IC95\%: 73,6-78,9). Os fatores independentes associados ao maior risco de óbito foram faixa etária menor de 30 anos (hazard ratio - HR = 3,09; IC95\%: 1,25-7,67); as mulheres analfabetas (HR =3,70; IC95\%: 1,44-9,55); as com estadiamento III (HR = 5,27; IC95\%: 2,56-10,82) e IV (HR =14,07; IC95\%: 6,81-29,06). Mulheres jovens são aquelas com piores taxas de sobrevida. Também existem muitas mulheres sendo diagnosticadas em estádios avançados, tendo uma sobrevida pior, demonstrando a necessidade de ações de diagnóstico precoce.

Neoplasias da Mama; Registros Hospitalares; Análise de Sobrevida; Saúde da Mulher

\section{Referências}

1. World Health Organization. The Breast Health Global Initiative (BHGI). http://www.who.int/cancer/ bghi.pdf (acessado em 10/Abr/2006).

2. Molina L, Dalben I, De Luca LA. Análise das oportunidades de diagnóstico precoce para as neoplasias malignas de mama. Rev Assoc Med Bras 2003; 49:185-90.

3. Instituto Nacional de Câncer. Estimativa 2008: incidência de câncer no Brasil. http://www.inca.gov. br/estimativa/2008/versaofinal.pdf (acessado em 24/Fev/2008).

4. Parkin DM, Bray F, Ferlay J, Pisani P. Global cancer statistics, 2002. CA Cancer J Clin 2005; 55:74-108.

5. Bland KI, Menck HR, Scott-Conner CE, Morrow M, Winchester DJ, Winchester DP. The National Cancer Data Base 10-year survey of breast carcinoma treatment at hospitals in the United States. Cancer 1998; 83:1262-73.

\section{Colaboradores}

I. J. C. Schneider e E. d'Orsi participaram de todas as etapas do estudo e da elaboração do artigo, incluindo a organização dos dados com base nos bancos de dados originais, análise dos dados, discussão e revisão final.

\section{Agradecimentos}

Às registradoras do registro hospitalar de câncer do Centro de Pesquisas Oncológicas de Santa Catarina e do Hospital de Caridade - Irmandade Nosso Senhor dos Passos, e à coordenação e funcionários do Sistema de Informação de Mortalidade do Estado de Santa Catarina, sem os quais este trabalho não poderia ter sido realizado.
6. Eifel P, Axelson JA, Costa J, Crowley J, Curran Jr. WJ, Deshler A, et al. National Institutes of Health Consensus Development Conference Statement: adjuvant therapy for breast cancer, November 1-3, 2000. J Natl Cancer Inst 2001; 93:979-89.

7. Lagerlund M, Bellocco R, Karlsson P, Tejler G, Lambe M. Socio-economic factors and breast cancer survival: a population-based cohort study (Sweden). Cancer Causes Control 2005; 16:419-30.

8. Field TS, Buist DS, Doubeni C, Enger S, Fouayzi H, Hart G, et al. Disparities and survival among breast cancer patients. J Natl Cancer Inst Monogr 2005:88-95.

9. Moraes AB, Zanini RR, Turchiello MS, Riboldi J, Medeiros LR. Estudo da sobrevida de pacientes com câncer de mama atendidas no hospital da Universidade Federal de Santa Maria, Rio Grande do Sul, Brasil. Cad Saúde Pública 2006; 22:2219-28. 
10. Bustamante-Teixeira MT, Faerstein E, Latorre MR Técnicas de análise de sobrevida. Cad Saúde Pública 2002; 18:579-94.

11. Traebert EE. Câncer de mama em mulheres com idade igual ou superior a 65 anos: estudo de probabilidade de sobrevida livre de doença [Dissertação de Mestrado]. Florianópolis: Universidade Federal de Santa Catarina; 2006.

12. Crippa CG, d'Acampora AJ, Araújo EJ, Marasciulo AC, Hallal ALC, Gondin G. Câncer de mama em mulheres jovens: um estudo de probabilidade de sobrevida livre de doença. Rev Bras Mastologia 2002; 12:23-8.

13. Szklo M, Nieto FJ. Epidemiology: beyond the basics. Maryland: Aspen Publishers Inc.; 2000.

14. Instituto Nacional de Câncer. Registros Hospitalares de câncer: rotinas e procedimentos. Rio de Janeiro: Instituto Nacional de Câncer; 2000.

15. Serrano TRG. Avaliação do Programa Estadual de Controle do Câncer de Colo Uterino e de Mama em Santa Catarina [Dissertação de Mestrado]. Florianópolis: Universidade Federal de Santa Catarina; 2002.

16. Camargo Jr. KR, Coeli CM. Reclink: aplicativo para o relacionamento de bases de dados, implementando o método probabilistic record linkage. Cad Saúde Pública 2000; 16:439-47.

17. Secretaria de Vigilância em Saúde, Ministério da Saúde. Sistema Nacional de Vigilância em Saúde: relatório de situação, Santa Catarina, 2005. http:// bvsms.saude.gov.br/bvs/publicacoes/caderno_sc_ 2007.pdf (acessado em 17/Mar/2008).

18. Carvalho MS, Andreozzi VL, Codeço CT, Barbosa MTS, Shimakura SE. Análise de sobrevida: teoria e aplicações em saúde. Rio de Janeiro: Editora Fiocruz; 2005.

19. Rapiti E, Fioretta G, Verkooijen HM, Vlastos G, Schafer P, Sappino AP, et al. Survival of young and older breast cancer patients in Geneva from 1990 to 2001. Eur J Cancer 2005; 41:1446-52.

20. Mendonça GAS, Silva AM, Caula WM. Características tumorais e sobrevida de cinco anos em pacientes com câncer de mama admitidas no Instituto Nacional de Câncer, Rio de Janeiro, Brasil. Cad Saúde Pública 2004; 20:1232-9.

21. Abreu E. Câncer de mama feminina em Goiânia: análise de sobrevida em dez anos na coorte diagnosticada em 1988-90 [Tese de Doutorado]. Rio de Janeiro: Escola Nacional de Saúde Pública, Fundação Oswaldo Cruz; 2002.

22. Pereira WMM. Mortalidade e sobrevida por câncer de mama, no Estado do Pará [Dissertação de Mestrado]. Rio de Janeiro: Escola Nacional de Saúde Pública, Fundação Oswaldo Cruz; 2001.

23. American Cancer Society. Cancer facts \& figures 2008. Atlanta: American Cancer Society; 2008.
24. Abreu E, Koifman S. Fatores prognósticos no câncer de mama feminino. Rev Bras Cancerol 2002; 48:113-31.

25. Jemal A, Siegel R, Ward E, Murray T, Xu J, Thun MJ. Cancer statistics, 2007. CA Cancer J Clin 2007; 57:43-66.

26. Cianfrocca M, Goldstein LJ. Prognostic and predictive factors in early-stage breast cancer. Oncologist 2004; 9:606-16.

27. Osborne C, Ostir GV, Du X, Peek MK, Goodwin JS The influence of marital status on the stage at diagnosis, treatment, and survival of older women with breast cancer. Breast Cancer Res Treat 2005; 93:41-7.

28. American Cancer Society. Breast cancer facts \& figures 2007-2008. Atlanta: American Cancer Society; 2007.

29. Brito C, Portela MC, Vasconcellos MTL. Assistência oncológica pelo SUS a mulheres com câncer de mama no Estado do Rio de Janeiro. Rev Saúde Pública 2005; 39:874-81.

30. Thuler LCS, Mendonça GA. Estadiamento inicial dos casos de câncer de mama e colo do útero em mulheres brasileiras. Rev Bras Ginecol Obstet 2005; 27:656-60.

31. Instituto Nacional de Câncer. Controle do câncer de mama: documento de consenso. http://www. inca.gov.br/publicacoes/Consensointegra.pdf (acessado em 20/Abr/2006).

32. Norman SA, Russell Localio A, Weber AL, Coates RJ, Zhou L, Bernstein L, et al. Protection of mammography screening against death from breast cancer in women aged 40-64 years. Cancer Causes Control 2007; 18:909-18.

33. Swedish Organised Service Screening Evaluation Group. Reduction in breast cancer mortality from organized service screening with mammography: 1. Further confirmation with extended data. Cancer Epidemiol Biomarkers Prev 2006; 15:45-51.

34. Malmgren JA, Atwood MK, Kaplan HG. Increase in mammography detected breast cancer over time at a community based regional cancer center: a longitudinal cohort study 1990-2005. BMC Cancer 2008; 8:131.

35. Anderson BO, Shyyan R, Eniu A, Smith RA, Yip CH, Bese NS, et al. Breast cancer in limited-resource countries: an overview of the Breast Health Global Initiative 2005 guidelines. Breast J 2006; 12 Suppl 1: S3-15.

36. Kemp C, Elias S, Gebrim LH, Nazário ACP, Baracat EC, Lima GR. Estimativa de custo do rastreamento mamográfico em mulheres no climatério. Rev Bras Ginecol Obstet 2005; 27:415-20.

Recebido em 19/Ago/2008

Versão final reapresentada em 11/Dez/2008 Aprovado em 26/Jan/2009 